\title{
MASS EXCHANGE BETWEEN STARS AND THE INTERSTELLAR GAS
}

\author{
BY LUDWIG BIERMANN
}

Star formation is most probably still going on in dense clouds of interstellar matter, either by direct condensation or by accretion of matter by stars of originally only moderate mass. There is even some indication, based on recent observations of stellar associations, that this process is going on at a rate which would cause it to exhaust the present amount of interstellar matter (probably of the order of $\frac{1}{2} \times 10^{10}$ solar masses) in a time comparable to, if not rather shorter than, the cosmic time scale of 5 billion years.

On the other hand, there are also observations which show that some types of stars lose mass to the interstellar material quite fast, and again a conservative estimate of the probable mass loss of the bulk of the normal, not particularly active, stars shows that in the life-time of our galactic system the losses may well add up to an amount comparable with, if not larger than, the present amount of interstellar material mentioned before.

These processes give a ready interpretation of the observed close correlation of the occurrence in our own, as well as in other galactic systems, of interstellar matter and those stars, the young age of which can be inferred from the rapid expenditure of their store of subatomic energy.

Furthermore, the possibility seems to emerge that the gains and losses of the interstellar material partly, if not mainly, balance each other. This possibility is interesting from still other points of view. The youngest stars appear to be richer in their content of certain elements, possibly formed when this material belonged to other stars a long time ago. The question whether or not the mass of a star varies in the course of the evolution has naturally a bearing on the theory of stellar structure. Certain observed properties of some classes of binary stars, studied especially by $O$. Struve, seem to call for an interpretation on this basis. Also the existence of the very numerous white dwarf stars, which should have reached a final state of evolution, can probably be understood only by the assumption that originally they had a mass of about two or more solar masses, whereas their present mass is much smaller.

Max Planck Institut,
Göttingen, Germany.

\section{ABSTRACTS*}

Abbot, C. G. Periodic solar variations.

There exists a family of periodic regular variations, all aliquot parts of $22 \frac{3}{4}$ years. Over 40 members of this family are known, some in the solar radiation, some in precipitation and in temperature on earth, some in basal pulse rates. All are, to within less than one per cent, exact submultiples of $22 \frac{3}{4} \times 365.2564$ days. Thus, in I948, I discovered periods of 6.6485 and 3.3242 days in New York and Washington weather, and I $/ \mathbf{1} 25^{0} \times 22 \frac{3}{4} \times 365.2564=6.6476$ days.

I have just completed a study of Smithsonian solar constant observations from September 1923 to December I952. As the published probable error of the mean of one day's measures from several stations is below $\frac{1}{8}$ per cent, the probable error of the mean values from 9 columns of roday means is below $\mathrm{I} / 75$ per cent. Hence it is possible not only to detect the stronger periods in solar variation, such as $30 \frac{1}{3}, 34 \frac{1}{5}, 39$ months, and other longer periods, but also many shorter periods, with ranges not exceeding $\mathrm{I} / 20$ per cent of the solar constant. Over 30 periods have been evaluated in solar variation.

This discovery obviously has a theoretical significance for the sun's constitution and for physiology as well as a practical significance for meteorology. In weather the individual periodicities range from 5 to 25 per cent of normal precipitation, and up to $2^{\circ}$ Fahrenheit in temperature.

Smithsonian Institution, Washington, D. C.

Adel, Arthur. The cyclical variations and the nonperiodic fine structure of ERTOR, effective radiation temperature of the ozone region.

The Atmospheric Research Observatory is engaged in a study of the infrared parameters of the earth's atmosphere. One of these is the effective radiation temperature of the ozone re-

* Of papers presented at the Ninety-second Meeting of the American Astronomical Society, Princeton, New Jersey, April 3-6, 1955 . 